\title{
Species Composition and Diversity of Mites Inhabiting Pomegranate Orchards at Assiut, Upper Egypt
}

\author{
Abdelgayed $^{*}$ S. A.; Abd El-Wahed"; N. M., Ali**; A. M. and Eraky ${ }^{* *}$, S. A. \\ * Plant Protec. Res. Institute, Agric. Res. Cent. Dokki, Giza, Egypt, ahmed.abdelgid@yahoo.com. \\ ** Department of Plant Protection, Faculty of Agriculture, Assiut University, Assiut 71526 Egypt.
}

\begin{abstract}
Mites play an important role in the biological cycle of nature, as well as in the agricultural cycles of plants. They are considered to be very important for most plants, especially fruit orchards, which case both, qualitative and quantitative losses, they also cause different types of allergenic reactions among the fruit handles and farmers. A comprehensive survey of mites inhabiting pomegranate orchards at Assiut and its suburbs was conducted in the work herein. During the current work, colonies of the pomegranate false spider mite, Tenuipalpus punicae Pritchard \& Baker and the mango spider mite, Oligonychus mangiferus (Rahman \& Sapra) were recorded on pomegranate trees at Assiut governorate. The relative abundance of both mite species and their natural enemies was conducted. Based on previous studies, about 24 mite species in nine families were recorded in Egypt. In order to determine species composition of phytophagous and predacious mites on pomegranate trees, samples were taken from leaves, buds and fruits during 2018/2019 and 2019/2020 seasons. The citrus brown mite, Eutetranychus orientalis (Klein); T. punicae and the broad mite, Polyphagotarsonemus latus (Banks) seemed to be the most common phytophagous mites on pomegranate trees. On the other side, several predatory mite species were also recorded in the present work. Of these, Amblyseius swirskii Athias-Henriot; Euseius scutalis (Athias-Henriot); Typhlodromus (Typhlodromus) pyri Scheuten; Neoseiulus barkeri Hughes (Phytoseiidae), and Agistemus exsertus Gonzalez (Stigmaeidae), all were recorded in large numbers, especially on pomegranate leaves of all varieties. While Cheletogenes ornatus (Canestrini \& Fanzago) and Cheletomimus bakeri (Ehara) (Cheyletidae) were recorded mostly on leaves and fruits. Quantifying the occurrence of various predacious mite species on pomegranate trees, may be of great importance to reduce the number of phytophagous ones. In separated subsequent studies, the efficacy of these predatory mites can be estimated as one of the biological control agents.
\end{abstract}

Key words: Acari; species composition; diversity; mites; pomegranate; Egypt.

\section{INTRODUCTION}

Pomegranate (Punica granatum L.) (Lythraceae) comprises about 500 cultivars worldwide (Stover and Mercure, 2007). It differs from all fruits in having pink small flowers and less sweet fruits. Pomegranate is used as fresh fruits or in baking, beverages and cooking. Moreover, it is largely used for healing from several diseases across different cultures and civilizations (Bhowmik et al., 2013). It has been mentioned many times in Holly Quran. Extracts of different parts of pomegranate plants and fruits have several nutritive, industrial, medicinal values and some pharmacological properties, where the extract contains hypertensive, antispasmodic and anthelmintic properties as well. The pomegranate orchards are liable to be attacked by several mite pests in Egypt, such as: the pomegranate flat mite, Brevipalpus lewisi (McGregor); the pomegranate false spider mite, Tenuipalpus punicae Pritchard \& Baker and the pomegranate eriophyid mite, Aceria granati (Canestrini \& Massalongo).Those pests attacking pomegranates, and can make holes and bores in the fruits, feeding on the berries to attract other mites and fungi. The essential taxonomic studies of agricultural mites in Egypt are extremely poor resulting in insufficient information concerning the biology and ecology of plant feeders and predatory mites (Zaher and El-Badry, 1962; Mohamed, 1963; Rasmy et al., 1972; Mohamed, 2018; Desokey et al., 2020). Such information are required for successful Integrated Pest Management (IPM) programs. Moreover, these information will help improvement of the recently introduced programs to Egypt such as organic farming and biological control (Saleh et al., 2015; Adly, 2016; Abdelgayed et al., 2017; Elhalawany et al., 2017). A few numbers of phytophagous mite species had been previously reported on pomegranate trees in Egypt (Zaher et al., 1971; Rasmy et al., 1972; Zaher and Yosef, 1972). Yet, no predatory mites on pomegranate trees had been recorded. However, some predatory mites had been found on different plants other than on pomegranate (Zaher et al., 1971; Rasmy et al., 1972; Zaher and Yosef, 1972; Zaher, 1984a,b; 1986; AboShnaf, 2005; Abo-Shnaf and Moraes, 2014; Abdelgayed, 2017). However, the previously mentioned studies did not provide enough information concerning either the description or the distribution of these mites. In addition, neither illustrated keys nor deposited specimens were available. Therefore, the objectives of the present work were to investigate phytophagous and predacious mite species associated with seven varieties of pomegranate trees covering different localities at Assiut governorate and its suburbs, and to take notes on occurrence period and distribution of such mites on different plants and localities. The identified mites presented in this work can be helpful in further investigations concerning mite fauna in Egypt. 


\section{MATERIALS AND METHODS}

Samples were taken during October 2018 till May 2020, in 11 districts at Assiut Governorate. Mites occurred on plant parts (i.e., leaves, fruits, branches, buds) of different varieties of Punica granatum L. (such as: Araby, Assiuty, Manfalouty, Hegazy, Nab El-Gamal, Tafiiy and Wardy), in addition to Bermuda grass, Cynodon dactylon L. (Poaceae) grown under the pomegranate trees. Mites were collected by direct observation under a stereoscopic microscope and by using specialized hand-held aspirators (BioQuip ${ }^{\circledR}$, CA, USA). Soon after, the plant materials were put in Berlese's funnels. The extracted mites were cleared in lactic acid, mounted on microscopic slides using Hoyer's medium and examined under (BH-2, Olympus ${ }^{\circledR}$, Japan) research microscope. Mites were identified using the world taxonomic literature. The voucher specimens were deposited in the Acari collection of the Department of Plant Protection, Faculty of Agriculture, Assiut University, Egypt.

\section{RESULTS AND DISCUSSION}

The present work showed the presence of 175 mite species belonging to 48 families on pomegranate trees at Assiut Governorate, Upper Egypt (Table 1). Of these, twenty-seven phytophagous mite species pertaining to three families (i.e., Tarsonemidae, Tenuipalpidae and Tetranychidae); ninety-two mite species in 22 families composed mostly of predaceous mites (Amroseiidae, Anystidae, Ascidae, Bdellidae, Blattisociidae, Cheyletidae, Cunaxidae, Digmasellidae, Erythraidae, Eupalopsellidae, Halolaelapidae, Laelapidae, Macrochelidae, Melicharidae, Ologamasidae, Pachylaelapidae, Parasitidae, Phytoseiidae, Sejidae, Stigmaeidae, Uropodidae and Veigaiidae), and 57 species in 23 families of diverse feeding habits (Acaridae, Brachychthonidae, Caligonellidae, Camerobiidae, Ereynetidae, Eupodidae, Galumnidae, Hemisarcoptidae, Histiostomatidae, Hypochthoniidae, Iolinidae, Lohmannidae, Microtrmbidiidae, Nothridae, Oplitidae, Oppiidae, Oribatulidae Pygmophoridae, Scheloribatidae, Scutacaridae, Trombiculidae, Trombididae and Tydeidae).The pomegranate false red mite, Tenuipalpus punicae Pritchard \& Baker, primarily a pest of pomegranate trees and reported from many parts worldwide. This species was firstly reported in Egypt by Sayed (1942). It feeds on the upper and lower sides of pomegranate's leaves and on fruits close to the midrib. It widespread on pomegranate trees at Assiut Governorate (Abdelgayed et al., 2019). On the other hand, the grape false red mite, Tenuipalpus granati Sayed is a harmful pest on pomegranate and grape-vine orchards in globe including Egypt (Sayed, 1946; Zaher and El-Badry, 1964; Zaher et al., 1970, 1971; Rasmy et al., 1972; Zaher and Yosef, 1972; Wahba et al., 1982).

The citrus brown mite, Eutetranychus orientalis (Klein), was a second harmful pest of pomegranate, it was reported from many parts of the world and it was firstly recorded in Egypt by Sayed (1942), this species prefers feeding on upper side of leaves close to the midrib. It always found on citrus trees at Assiut Governorate (Salman et al., 1975). Certain pomegranate varities are liable to be severely infested with citrus brown mite. The broad mite, Polyphagotarsonemus latus (Banks), seemed to be an important pest on pomegranate trees cultivated in both Abou-Teig and Assiut cities, based on its high densities and subsequent discoloration of leaves. The phytophagous flat mites, Cenopalpuspulcher (Canestrini \& Fanzago); Phyllotetranychus aegyptiacus Sayedand T. eriophyoides Baker were also infested pomegranate leaves, but in moderate at Assiut districts. Eraky (1994) described the new mite species, Histiostoma rizkii on pomegranate fruits at Assiut Governorate.

The surveyed predacious mite species recorded on pomegranate trees were belonging to the families: Phytoseiidae, Stigmaeidae and Cheyletidae. The Amblyseius swirskii; E. scutalis and T. (T.) pyri were the most numerous phytoseiid species commonly found on pomegranate leaves of the pomegranate varieties: Assiuty, Araby and Manfalouty "Baladi", searching on their suitable prey (i.e., E. orientalis; $O$. mangiferus and $T$. punicae).The Neoseiulus barkeri was found on Bermuda grass, C. dactylon directly in association with the pomegranate whitefly, Siphoninus phillyreae (Haliday); the pomegranate aphid, Aphis punicae Passerini and the grape thrips, Rhipiphorothrips cruentatus Hood. The E. scutalis was recorded as predator on both E. orientalis and Brevipalpus obovatus Donnadieuin (Donia et al., 1995), they may work well against T. punicae. Among other recorded predators, Cheletogenes ornatus and Cheletomimus bakeri were found in high numbers on pomegranate leaves and fruits of pomegranate varieties (i.e., Araby and Manfalouty). Also, the Cheletomimus bakeri was recorded feeding on citrus red mite, Panonychus citri (McGregor) in Ehime prefecture of Japan (Razaq et al., 2001), this indicated that, this species may act well against $T$. punicae. On the other side, Mesbah and Omer (2014) studied the life-table parameters of Cheletogenes ornatus reared on the red palm mite, Raoiella indica and date scale-insect, Parlatoria blanchardii (Targ.). Also, Zaher et al. (1981); and Moraes et al. (1989) recorded that, Cheletogenes ornatus was noticed to be associated with tenuipalpid mites. 
Table (1). List of mite species reported from pomegranate orchards at Assiut, Upper Egypt

\begin{tabular}{|c|c|c|c|}
\hline Mite taxa & Varieties of pomegranate & Locality* & Incidence** \\
\hline \multicolumn{4}{|l|}{ Acaridae Latreille } \\
\hline Acotyledon manuri Eraky & Assiuty, Manfalouty (Baladi) & 1 & +++ \\
\hline A. nerminka Eraky & Assiuty, Manfalouty, Wordy & 1 & ++ \\
\hline Caloglyphus citriEraky et al. & Araby, Nab El-Gamal & 2,3 & + \\
\hline Cosmoglyphus manuri Negm & Manfalouty, Wordy & 1,2 & + \\
\hline Forcellinia mahunkai Eraky & Araby & 1 & ++ \\
\hline Rhizoglyphus echinopus (Fumouze \& Robin) & Manfalouty & 1 & + \\
\hline R. robiniClaparède & Assiuty, Wordy & 1 & + \\
\hline \multicolumn{4}{|l|}{ Ameroseiidae Evans } \\
\hline Ameroseius aegypticusEl-Badry, Nasr \& Hafez & Araby, Nab El-Gamel & 1 & ++ \\
\hline Kleemannia kosi El-Badry, Nasr \& Hafez & Assiuty, Mafalouty & $1,2,10,11$ & ++ \\
\hline K. nova Nasr \& Abou-Awad & Assiuty & 1,2 & ++ \\
\hline K. parplumosaNasr \& Abou-Awad & Assiuty & 1 & ++ \\
\hline K. plumosa (Oudemans) & Araby, Mafalouty & 1,2 & +++ \\
\hline Sertitympanumaegyptiacum Nasr \& Abou-Awad & Manfalouty & 2,11 & +++ \\
\hline S.zaheri(El-Badry, Nasr \& Hafez) & Assiuty & 9 & + \\
\hline \multicolumn{4}{|l|}{ Anystidae Oudemans } \\
\hline Anystis baccarum Linnaeus & Araby & $1,2,10$ & + \\
\hline Paratarsotomusmacropalpis (Banks) & Nab El-Gamal & 1 & + \\
\hline \multicolumn{4}{|l|}{ Ascidae Voigts \& Oudemans } \\
\hline Arctoseius bilinear Nasr & Nab El-Gamel & 1 & + \\
\hline Gamasellodes bicolor (Berlese) & $\begin{array}{l}\text { Assiuty, Hegazy, Mafalouty, Nab El- } \\
\text { Gamel }\end{array}$ & $1,2,8$ & ++ \\
\hline G. zaheri Nasr & Manfalouty, Nab El-Gamel & 1,2 & + \\
\hline Protogamasellus denticus Nasr & Assiuty & 1,2 & + \\
\hline P. minutus Nasr & Manfalouty & 2 & + \\
\hline \multicolumn{4}{|l|}{ Bdellidae Dugès } \\
\hline Cyta latirostris (Hermann) & Manfalouty & 1 & + \\
\hline Spinibdella bifurcata Atyeo & Assiuty, Manfalouty & 4 & ++ \\
\hline \multicolumn{4}{|l|}{ Blattisociidae Garman } \\
\hline Blattisocius dentriticus (Berlese) & Assiuty & 1 & ++ \\
\hline B. keegani Fox & Manfalouty & 1 & + \\
\hline Lasioseiusafricanus Nasr & Hegazy, Mafalouty & 2 & + \\
\hline L. parberlesei Bhattacharyya & Manfalouty, Assiuty & 2,1 & + \\
\hline L. youcefi Athias-Henriot & Araby & 3 & + \\
\hline \multicolumn{4}{|l|}{ Brachychthonidae Polski } \\
\hline Brachychthonius amicabilis Gil-Martín \& Subías & Wordy & 1 & + \\
\hline B. pauliani Balogh \& Mahunka & Assiuty & 1 & ++ \\
\hline \multicolumn{4}{|l|}{ Caligonellidae Grandjean } \\
\hline Molothrognathus minutus Soliman & Araby & 2,5 & + \\
\hline \multicolumn{4}{|l|}{ Camerobiidae Southcott } \\
\hline Neophyllobius aegyptium Soliman \& Zaher & Manfalouty & 1 & + \\
\hline N. mangiferus Zaher \& Gomaa & Assiuty & $1,2,5$ & ++ \\
\hline \multicolumn{4}{|l|}{ Cheyletidae Leach } \\
\hline Acaropsella volgini (Gerson) & Araby, Assiuty, Hegazy, Wordy & 1 & + \\
\hline Cheletogenes ornatus (Canestrini \& Fanzago) & Assiuty, Manfalouty, Wordy & $1,2,4$ & +++ \\
\hline Cheletomimus bakeri (Ehara) & Assiuty, Araby, Hegazy, Manfalouty & 1,10 & + \\
\hline C. congensis (Cunliffe) & Assiuty, Araby, Hegazy, Manfalouty & $1,2,10$ & + \\
\hline Cheyletus cacahuamilpensisBaker & Assiuty & 1 & + \\
\hline C. malaccensis Oudemans & Manfalouty & 2 & + \\
\hline Cunliffella ornata (Wafa \& Soliman) & Araby, Nab El-Gamal & 1 & ++ \\
\hline \multicolumn{4}{|l|}{ Cunaxidae Thor } \\
\hline Coleoscirus simplex (Ewing) & Assiuty & 1,7 & + \\
\hline Cunaxa capreolus (Berlese) & Assiuty, Manfalouty & 1,4 & + \\
\hline C. setirostris (Hermann) & Manfalouty & 1 & + \\
\hline Cunaxoides croceus (Koch) & Manfalouty & 2 & + \\
\hline Dactyloscirus saopauloensis Den Heyer & Manfalouty & 10 & + \\
\hline Neocunaxoides ovatusLin, Zhang \& Ji & Wordy, Manfalouty, Nab El-Gamal & 1,4 & ++ \\
\hline
\end{tabular}


Table (1). Continue

\begin{tabular}{|c|c|c|c|}
\hline Mite taxa & Varieties of pomegranate & Locality* & Incidence** \\
\hline \multicolumn{4}{|l|}{ Digamasellidae Evans } \\
\hline Dendrolaelaps aegypticus Metwally \& Mersal, 1978 & Araby & 1 & + \\
\hline D. zaheri Metwally \& Mersal & Manfalouty, Wordy & 1,4 & ++ \\
\hline \multicolumn{4}{|l|}{ Ereynetidae Oudemans } \\
\hline Ereynetes bolitophilus Fain\& Camerik & Assiuty, Wordy & 1 & + \\
\hline \multicolumn{4}{|l|}{ Erythraeidae Oudemans } \\
\hline Erythraeus berninensis Haitlinger & Wordy & 1 & + \\
\hline \multicolumn{4}{|l|}{ Eupalopsellidae Willmann } \\
\hline Eupalopsellus olearius Zaher \& Gomaa & Manfalouty & $4,6,8,11$ & + \\
\hline \multicolumn{4}{|l|}{ Eupodidae Koch } \\
\hline Eupodesniloticus Abou-Awad \& El-Bagoury & Araby, Manfalouty, Nab El-Gamal & $1,2,9,10$ & + \\
\hline \multicolumn{4}{|l|}{ Galumnidae Jacot } \\
\hline Pilogalumna ornatula Grandjean & Araby & 1 & + \\
\hline \multicolumn{4}{|l|}{ Halolaelapidae Karg } \\
\hline Halolaelaps sexclavatus (Oudemans) & Araby, Manfalouty & $1,2,8$ & + \\
\hline \multicolumn{4}{|l|}{ Hemisarcoptidae Oudemans } \\
\hline Hemisarcoptes malus (Shimer) & Araby & 1 & + \\
\hline \multicolumn{4}{|l|}{ Histiostomatidae Berlese } \\
\hline Copronomia mahunkai Eraky & $\begin{array}{l}\text { Assiuty, Araby, Wordy, Manfalouty, } \\
\text { Nab El-Gamal }\end{array}$ & $1,3,8$ & +++ \\
\hline Histiostomaarcuatus Negm & Araby, Hegazy & 1 & ++ \\
\hline H. camphori Eraky & Hegazy & 1 & + \\
\hline H. darwishii Eraky & Assiuty, Araby, Hegazy, Manfalouty & 1,2 & +++ \\
\hline H. nasseri Eraky & Assiuty & 1 & + \\
\hline H. onioni Eraky \& Shoker & Nab El-Gamal & 1 & + \\
\hline H. rizkii Eraky & Assiuty, Araby. & 1,2 & + \\
\hline Myianoetus lili Eraky & Manfalouty & 1 & + \\
\hline \multicolumn{4}{|l|}{ Hypochthoniidae Berlese } \\
\hline Hypochthoniusrufulus (Koch) & Araby, Assiuty & 1 & + \\
\hline \multicolumn{4}{|l|}{ Iolinidae Pritchard } \\
\hline Pronematus ubiquitus (McGregor) & Manfalouty & 1,4 & ++ \\
\hline \multicolumn{4}{|l|}{ Laelapidae Berlese } \\
\hline Androlaelaps aegypticus Hafez, El-Badry \& Nasr & Wordy & 1 & + \\
\hline A. casalis (Berlese, 1887) & Assiuty & $2,3,6$ & + \\
\hline A. zaheri (Hafez, El-Badry \& Nasr) & Wordy & 1 & + \\
\hline Cosmolaelaps longus (Hafez, El- Badry \& Nasr) & Wordy & $2,4,5$ & ++ \\
\hline Gaeolaelaps bregetovae (Shereef \& Afifi) & Manfalouty & 2 & ++ \\
\hline G. orientalis (Hafez, El-Badry \& Nasr) & Araby & 2 & + \\
\hline G. queenslandicus (Womersley) & Wordy & 4 & + \\
\hline Hypoaspis wohabi Metwally \& Ibrahium & Araby, Wordy & $1,2,3$ & ++ \\
\hline Laelaspis astronomicus (Koch) & Araby & 9 & ++ \\
\hline Ololaelasps bregetovae Shereef \& Soliman & $\begin{array}{l}\text { Araby, Manfalouty, Nab El-Gamal, } \\
\text { Wordy }\end{array}$ & 1,2 & +++ \\
\hline \multicolumn{4}{|l|}{ Lohmanniidae Berlese } \\
\hline Lohmannia egypticus (El-Badry \& Nasr) & Assiuty & 1 & +++ \\
\hline Papillacarus aciculatus Kunst & Araby & 1 & + \\
\hline \multicolumn{4}{|l|}{ Macrochelidae Vitzthum } \\
\hline Macrocheles lagodekhensis Bregetova \& Koroleva & Assiuty & 1 & + \\
\hline M. merdarius (Berlese) & Assiuty & 1 & + \\
\hline M.muscaedomesticae (Scopoli) & Assiuty & 1 & ++ \\
\hline M. perglaber Filipponi \& Pegazzano & Wordy & 1 & + \\
\hline M. solimani Hafez, El-Badry \& Nasr & Assiuty & 1,9 & + \\
\hline M. submotus (=M. congnatus) Falconer & Assiuty & 1 & + \\
\hline \multicolumn{4}{|l|}{ Melicharidae Hirschmann } \\
\hline Proctolaelaps aegyptiacus Nasr & Manfalouty & 3,4 & ++ \\
\hline P. afifii Moraes, Britto, Mineiro \& Halliday & Manfalouty & 4 & + \\
\hline P. holoventris Moraes, Britto, Mineiro \& Halliday & Assuity & $1,2,5,6$ & +++ \\
\hline P. orientalis Bhattacharyya & Hegazy, Manfalouty & 1,4 & + \\
\hline P. pygmaeus (Mülle) & Araby, Manfalouty, Nab El-Gamel & $1,2,3,4$ & +++ \\
\hline P. scolyti Evans & Araby & 1 & + \\
\hline
\end{tabular}


Table (1). Continue

\begin{tabular}{|c|c|c|c|}
\hline Mite taxa & Varieties of pomegranate & Locality* & Incidence** \\
\hline \multicolumn{4}{|l|}{ Macrochelidae Vitzthum } \\
\hline Macrocheles lagodekhensis Bregetova \& Koroleva & Assiuty & 1 & + \\
\hline M. merdarius (Berlese) & Assiuty & 1 & + \\
\hline M.muscaedomesticae (Scopoli) & Assiuty & 1 & ++ \\
\hline M. perglaber Filipponi \& Pegazzano & Wordy & 1 & + \\
\hline M. solimani Hafez, El-Badry \& Nasr & Assiuty & 1,9 & + \\
\hline M. submotus (=M. congnatus) Falconer & Assiuty & 1 & + \\
\hline \multicolumn{4}{|l|}{ Melicharidae Hirschmann } \\
\hline Proctolaelaps aegyptiacus Nasr & Manfalouty & 3,4 & ++ \\
\hline P. afifii Moraes, Britto, Mineiro \& Halliday & Manfalouty & 4 & + \\
\hline P. holoventris Moraes, Britto, Mineiro \& Halliday & Assuity & $1,2,5,6$ & +++ \\
\hline P. orientalis Bhattacharyya & Hegazy, Manfalouty & 1,4 & + \\
\hline P. pygmaeus (Mülle) & Araby, Manfalouty, Nab El-Gamel & $1,2,3,4$ & +++ \\
\hline P. scolyti Evans & Araby & 1 & + \\
\hline \multicolumn{4}{|l|}{ Microtrombidiidae Thor } \\
\hline Microtrombidium pusillum (Hermann) & Assiuty & 1 & ++ \\
\hline \multicolumn{4}{|l|}{ Nothridae Berlese } \\
\hline Nothrus anauniensis Canestrini \& Fanzago & Assiuty & 1 & ++ \\
\hline \multicolumn{4}{|l|}{ Ologamasidae Ryke } \\
\hline Gamasiphis denticus Hafez \& Nasr & Assiuty, Mafalouty & 1 & + \\
\hline G. parpulchellus Nasr \& Mersal & Assiuty, Manfalouty & 2,6 & ++ \\
\hline G. pulchellus (Berlese) & Araby & $1,2,3,4$ & +++ \\
\hline \multicolumn{4}{|l|}{ Oplitidae Johnston } \\
\hline Oplitis minutissima (Berlese) & Araby & 9 & ++ \\
\hline \multicolumn{4}{|l|}{ Oppiidae Grandjean } \\
\hline Multioppia wilsoni Akoi & Manfalouty & $1,3,8,11$ & + \\
\hline Oppia inclinata Hammer & Araby & 1 & + \\
\hline \multicolumn{4}{|l|}{ Oribatulidae Thor } \\
\hline Scheloribates zaheri Youssef \& Nasr & Manfalouty & 6,9 & + \\
\hline Siculobata sicula (Berlese) & Manfalouty & 1,2 & ++ \\
\hline Zygoribatula tadrosi (Popp) & Araby & 1 & + \\
\hline Z. tameyai El-Badry \& Nasr & Manfalouty & 1 & + \\
\hline Z. sayedi El-Badry \& Nasr & Assiuty & 1,2 & ++ \\
\hline \multicolumn{4}{|l|}{ Pachylaelapidae Berlese } \\
\hline Pachylaelapsaegyptiacus Hafez \& Nasr & Assiuty & 9 & + \\
\hline Zygoseius furciger (Berlese) & Assiuty, Manfalouty, Wordy & $1,4,8,9$ & ++ \\
\hline \multicolumn{4}{|l|}{ Parasitidae Oudemans } \\
\hline Parasitus badrii Hafez \& Nasr & Araby & 2 & + \\
\hline P. consanguineus Oudemans \& Voigts & Assiuty, Manfalouty & $1,3,4,9$ & +++ \\
\hline$P$. fimetorum (Berlese) & Assiuty, Araby & $1,2,3,9$ & ++ \\
\hline P. hyalinus (Willmann) & Manfalouty & 1 & + \\
\hline P. insignis Holzmann) & Assiuty & 1 & + \\
\hline P. kempersi Oudemans & Assiuty & 1 & + \\
\hline P loricatus (Wankel) & Manfalouty & 1 & + \\
\hline \multicolumn{4}{|l|}{ Phytoseiidae Berlese } \\
\hline Amblyseius swirskii Athias-Henriot & Assiuty, Manfalouty, Wordy & 1,2 & ++ \\
\hline Cydnoseius negevi (Swirski \& Amitai) & Assiuty, Manfalouty & $1,10,11$ & + \\
\hline Euseius scutalis (Athias-Henriot) & Assiuty, Araby, Manfalouty & $1,2,3,4,5$ & +++ \\
\hline Neoseiulus barkeri Hughes & Araby, Hegazay, Manfalouty & $1,2,3,4,7$ & +++ \\
\hline N. mumae (Shehata \& Zaher) & Manfalouty & 4 & + \\
\hline Proprioseiopsis messor (Wainstein) & Assiuty, Araby, Nab EL-Gamal & $1,2,6,8$ & + \\
\hline P. ovatus (Garman) & Manfalouty & $1,2,6,9$ & + \\
\hline Typhlodromus (Typhlodromus) pyri Scheuten & Wordy & $1,2,3,6$ & ++ \\
\hline \multicolumn{4}{|l|}{ Pygmephoridae Cross } \\
\hline Bakerdania arvorum (Jacot) & Assiuty & 1,3 & + \\
\hline B. centriger (Cooreman) & Assiuty & 4,6 & + \\
\hline B. exigua (Mahunka) & Araby, Assiuty & 3,4 & + \\
\hline B. montana (Willmann) & Manfalouty & 1,2 & + \\
\hline B. punctata Zaher \& Kandeel & Manfalouty & 10,11 & ++ \\
\hline B. togata (Willmann) & Manfalouty & $5,8,9$ & + \\
\hline Pediculaster arabicus Zaher \& Kandeel & Manfalouty & 1,7 & ++ \\
\hline
\end{tabular}


Table (1). Continue

\begin{tabular}{|c|c|c|c|}
\hline Mite taxa & Varieties of pomegranate & Locality* & Incidence** \\
\hline \multicolumn{4}{|l|}{ Pygmephoridae Cross } \\
\hline P. demeterorum Mahunka & Araby & 3,9 & + \\
\hline P. endroedyi (Mahunka) & Assiuty & 11 & + \\
\hline P. ignotus Krczal & Manfalouty & 10 & + \\
\hline Pygmephorus stammeri Krczal & Manfalouty & 8 & + \\
\hline \multicolumn{4}{|l|}{ Scheloribatidae Jacot } \\
\hline Scheloribatespraeincisus (Berlese) & Manfalouty & 1 & + \\
\hline \multicolumn{4}{|l|}{ Scutacaridae Oudemans } \\
\hline Imparipes vulgaris Delfinado \& Baker & Manfalouty, Assiuty & 8 & + \\
\hline Scutacarus deficions Mahunka & Assiuty & 2 & ++ \\
\hline \multicolumn{4}{|l|}{ Sejidae Berlese } \\
\hline Sejus americanus(Banks) & Araby, Assiuty, NabEl-Gamel & 1,3 & + \\
\hline \multicolumn{4}{|l|}{ Stigmaeidae Oudemans } \\
\hline Agistemus exsertus Gonzalez & $\begin{array}{l}\text { Assiuty, Manufalouty, Wordy, } \\
\text { Araby }\end{array}$ & 1,11 & +++ \\
\hline A.vulgaris Soliman \& Gomaa & Hegazy & 2 & + \\
\hline \multicolumn{4}{|l|}{ Tarsonemidae Kramer } \\
\hline Polyphagotarsonemus latus ${ }^{1}$ (Banks) & Assiuty, Manfalouty & $1,2,10$ & ++ \\
\hline Tarsonemus bilobatus Suski & Manfalouty & 1 & ++ \\
\hline T. buxi (Canestrini \& Berlese) & Manfalouty & 4 & + \\
\hline T. fennicus Oudemanus & Assiuty & 7 & + \\
\hline T. lobosus Suski & Assiuty & 9 & + \\
\hline T. myceliophagus Hussey & Manfalouty, Assiuty & $1,2,3$ & ++ \\
\hline T. noxius (Humiczewska) & Manfalouty & 7 & + \\
\hline T. schaarschmiditi Mahunka & Wordy & 1 & + \\
\hline T. setifer Ewing & Araby & 1 & + \\
\hline T. stammeri Schaarschmidt & Assiuty & 4 & + \\
\hline T. subcorticalisLindquist & Araby, Assiuty & 1,2 & + \\
\hline T. talpae Schaarschmidt & Manfalouty & 8 & + \\
\hline T. virgineus Suski & Assiuty & 2 & + \\
\hline \multicolumn{4}{|l|}{ Tenuipalpidae Berlese } \\
\hline Brevipalpus phoenicis (Geijskes) & Assiuty, Manfalouty & $1,2,3,8$ & + \\
\hline Cenopalpus lanceolatisetae Attiah & Assiuty & 10 & + \\
\hline C. pulcher (Canestrini \& Fanzago) & Manfalouty & 2 & + \\
\hline Phyllotetranychus aegyptiacus Sayed & Assiuty & 1,10 & + (accidently). \\
\hline Raoiella indica Hirst & Assiuty & 1,10 & + (accidently). \\
\hline Tenuipalpus eriophyoides Baker & Manfalouty & 1 & + \\
\hline T. granati Sayed & Manfalouty & 2 & + \\
\hline T. punicae Pritchard \& Baker & Assiuty, Manfalouty, Wordy & $\begin{array}{l}1,2,3,4,5,6,7, \\
8,10,11\end{array}$ & +++ (cosmopolitan). \\
\hline \multicolumn{4}{|l|}{ Tetranychidae Donnadieu } \\
\hline Eutetranychus orientalis (Klein) & Assiuty & 1,7 & + \\
\hline E. palmatus Attiah & Assiuty, Manfalouty & 1,7 & + (accidently). \\
\hline Oligonychusafrasiaticus (McGregor) & Manfalouty & 2 & + (accidently). \\
\hline O. mangiferus (Rahman \& Sapra) & Assiuty, Manfalouty, Wordy & $1,3,8$ & +++ \\
\hline O. punicae ${ }^{1}$ (Hirst) & Assiuty & 1,3 & ++ \\
\hline Tetranychus urticae Koch & $\begin{array}{l}\text { Assiuty, Manfalouty, Hegazy, } \\
\text { Wardy, Al-Taify }\end{array}$ & $1,2,5$ & ++ \\
\hline \multicolumn{4}{|l|}{ Trombiculidae Ewing } \\
\hline Trombicula autumnalis (Shaw) & Manfalouty & 4 & + \\
\hline \multicolumn{4}{|l|}{ Trombidiidae Leach } \\
\hline Dolichothrombium anatoliae Mąkol \& Sevsay & Manfalouty & 5 & + \\
\hline Trombidium holosericeum(Linnaeus) & Assiuty & 1,6 & + \\
\hline \multicolumn{4}{|l|}{ Tydeidae Kramer } \\
\hline Tydeus californicus (Banks) & Nab El-Gamal, Wordy & 4 & + \\
\hline T. kochi (Oudemans) & Araby, Manfalouty & 1 & + \\
\hline \multicolumn{4}{|l|}{ Uropodidae Kramer } \\
\hline Urodiaspis aegypticus Ahmed & Assiuty, Manfalouty & $1,4,5,7,9$ & +++ \\
\hline Uroobovella ibiolis Vitzthum & Manfalouty & 1 & + \\
\hline U. krantzi (Zaher \& Afifi) & Manfalouty, Wordy & 1,2 & +++ \\
\hline U. marginata (Koch) & Assiuty & 1 & + \\
\hline Uropoda orbicularis (Müller) & Assiuty & 1,2 & ++ \\
\hline
\end{tabular}


Table (1). Continue

\begin{tabular}{|c|c|c|c|}
\hline Mite taxa & Varieties of pomegranate & Locality $^{*}$ & Incidence $^{* * *}$ \\
\hline \multicolumn{4}{|l|}{ VeigaiidaeOudemans } \\
\hline Cyrthydrolaelaps hirta Berlese & Assiuty & 1 & + \\
\hline Veigaia propinqua Willmann & Assiuty & 1 & + \\
\hline \multicolumn{4}{|l|}{ Trombiculidae Ewing } \\
\hline Trombicula autumnalis (Shaw) & Manfalouty & 4 & + \\
\hline \multicolumn{4}{|l|}{ Trombidiidae Leach } \\
\hline Dolichothrombium anatoliae Mąkol \& Sevsay & Manfalouty & 5 & + \\
\hline Trombidium holosericeum(Linnaeus) & Assiuty & 1,6 & + \\
\hline \multicolumn{4}{|l|}{ Tydeidae Kramer } \\
\hline Tydeus californicus (Banks) & Nab El-Gamal, Wordy & 4 & + \\
\hline T. kochi (Oudemans) & Araby, Manfalouty & 1 & + \\
\hline \multicolumn{4}{|l|}{ Uropodidae Kramer } \\
\hline Urodiaspis aegypticus Ahmed & Assiuty, Manfalouty & $1,4,5,7,9$ & +++ \\
\hline Uroobovella ibiolis Vitzthum & Manfalouty & 1 & + \\
\hline U. krantzi (Zaher \& Afifi) & Manfalouty, Wordy & 1,2 & +++ \\
\hline U. marginata (Koch) & Assiuty & 1 & + \\
\hline Uropoda orbicularis (Müller) & Assiuty & 1,2 & ++ \\
\hline \multicolumn{4}{|l|}{ VeigaiidaeOudemans } \\
\hline Cyrthydrolaelaps hirta Berlese & Assiuty & 1 & + \\
\hline Veigaia propinqua Willmann & Assiuty & 1 & + \\
\hline
\end{tabular}

In general, the current survey of mite species on pomegranate trees in Upper Egypt revealed the diversity of mite fauna (Phytophagous and predacious), which gives a balance between the population of each group of mites with noticeable increase in the number of predatory species, this may be due to the extensive use of pesticides to control both phytophagous mite and insect species hoping to obtain pesticide resistant strain of predatory mites.

\section{ACKNOWLEDGMENTS}

Thanks is to Prof. A.M. Metwally (Agric. Zoology and Nematology, Dept., Faculty of Agriculture, AlAzhar Universtity, Egypt) for identifying some mesostigmatid mite species. Thanks also to Prof. M.M. Kandeel (Faculty of Technology and Development, Zagazig Universty, Egypt) for confirming the identification of some tarsonemid species. Thanks also to pomegranate farmers for allowing to visit their pomegranate orchards and take samples.

\section{REFERENCES}

Abdelgayed, S. A. 2017. Species composition of phytophagous and predatory mites associated with citrus orchards in Assiut Governorate. M.Sc. Thesis, Faculty of Agriculture, Assiut University, Egypt, 155 pp.

Abdelgayed, S. A.; Abd El-Wahed, N. M.; Ali, A. M. and Eraky, S. A. 2019. Biological aspects, thermal requirements and life table parameters of Tenuipalpus punicae Pritchard \& Baker, 1958 (Acari: Tenuipalpidae) on pomegranate over different temperatures at Assiut, Egypt. Acarines,13: 21-27.

Abdelgayed, S. A.; Negm, M. W.; Eraky, S. A.; Helal, T. Y. and Moussa, S. F. M. 2017. Phytophagous and predatory mites inhabiting citrus trees at Assiut Governorate, Upper Egypt. Assiut J. Agric. Sci., 48 (1): 173-181.

Abo-Shnaf, R. I. A. 2005. Studies on some aerial and soil mites inhabiting fruit trees in Fayoum Governorate. M.Sc. Thesis, Faculty of Agriculture, Cairo Univ., El-Fayoum Branch, Egypt, 221 pp.

Abo-Shnaf, R. I. A. and Moraes, G. J. de. 2014. Phytoseiid mites (Acari: Phytoseiidae) from Egypt, with new records, descriptions of new species, and a key to species. Zootaxa, 3865 (1): 1-71.

Adly, D. 2016.Use of predators for controlling the whitefly, Bemisia tabaci Genn.and the Two spotted spider mite, Tetranychus urticae Koch., in Cucumber Greenhouses in Egypt. Egypt. J. Biol. Pest Cont., 26 (4): 701-706.

Bhowmik, D.; Gopinath, H.; Pragati Kumar, B.; Duraivel, S.; Aravind, G. and Sampath Kumar, K. P. 2013. Medicinal uses of Punica granatum and its health benefits. J. Pharmacognosy and Phytochemistry, 1 (5): 28-35.

Desoky, A. S. S.; Ahamed, H. E. M. and Eraky, S. A. 2020.Survey of mite species inhabiting animal production farm at Sohag Governorate, with checklist of mites existing manure and dung hills in Egypt. Int. J. Res. Agric. Foresty, 7 (4): 21-28. Donia, A. R. A.; Helal, E. M.; El-Hamid, A. A. and Zakzouk, E. A. 1995. Mass rearing and field evaluation of released predaceous mite, Euseius 
scutalis (Athias-Henriot) on the citrus whitefly, Aleurotrachelus citri (Priesner and Hosny). Alex. J. Agric. Res., 3: 209-219.

Elhalawany, A. S.; Abdel-Wahed, N. M. and Ahmed, N. F. R. 2017. Influence of prey type on the biology and life-Table parameters of Neoseiulus californicus (McGregor) (Acari: Phytoseiidae). Acarines, 11: 15-20.

Eraky, S. A. 1994. Two new hypopi of Histiostoma Kramer, 1876 (Acari: Astigmata) recovered from pomegranate and date fruits. Assiut J. Agric. Sci.,25 (2): 157-162.

Mesbah, A. E. and Omar, N. A. 2014. Predator-Prey Preferences and Life-Table-Parameters of Cheletogenes ornatus (Canestrini \&Fanzago) to Red Palm Mite Raoiella indica Hirst and Date Scale-Insect Parlatoria blanchardii (Targ.). Acarines, 8 (1): 19-23.

Mohamed, A. S. M. 2018. Ecological, biological and control studies on mites associated with some varieties of mango trees in Sohag and Assiut Governorates. M.Sc. Thesis, Al-Azhar University, Faculty of Agriculture, Egypt, 229 pp.

Mohamed, I. I. 1963. Mite pests in Egypt and their treatment. Egyptian Anglo Library, Almaarifa Press, 187 pp.

Moraes, G. J. de; Neto, R. S. and Pinto, H. C. S. 1989. Morphology, biology and pesticide tolerance of Chelotogenes ornatus (Acari: Cheyletidae). Entomophaga, 34 (4): 477-484.

Rasmy, A. H.; Zaher, M. A. and Abou-Awad, B. A. 1972. Mites associated with deciduous fruit trees in U.A.R. Z. ang. Ent., 70: 179-183.

Razaq, A.; Shiraishi, M.; Manabe, T. and Ohbayashi, N. 2001. External features of cheyletid predatory mite, Hemicheyletia bakeri (Ehara) found in citrus orchards of Japan. Pak. J. Biol. Sci., 4(5): 597601.

Saleh, E. B. Y., Mostafa, M. A., Desuky, W. M. H. and El-Kawas, H. M. G. 2015. Thermal units of the predatory mite, Euseius scutalis (AthisaHenriot) (Acari: Phytoseiidae) fed on crawlers of Bemisia tabaci (Genn.) (Hemiptera: Aleyrodidae). Egypt. J. Biol. Pest Cont., 25 (3): 683-687. In: Proc. 4th Int. Conf., ESPCP 2015, Cairo, Egypt, 19-22 October, 2015.

Salman, A. G. A.; Negm, A. A.; Abou-Ghadir, M. F.; Ali, A. M. and Darwesh, A. Y. 1975. Seasonal trends of citrus mites, Eutetranychus orientalis (Klein) and Brevipalpus phoenicis (Geijskes), and the role of predaceous mites, total nitrogen and total carbohydrates in relation to degree of infestation of citrus trees. Assiut J. Agric. Sci., 6 (4): $127-140$.
Sayed, M. T. 1942. Contribution to the knowledge of Acarina of Egypt. IV. The Genus Anychus McGregor [Tetranychidae]. Bull. Soc. Fouad I ${ }^{\mathrm{er}}$ Ent., 26: 125-131.

Sayed, M. T. 1946. The genus Anychus McGregor in Egypt and the Sudan [Acarina: Tetranychidae]. Bull. Soc. Fouad I ${ }^{\mathrm{er}}$ Ent. Eegypte, 30: 143-148.

Stover, E. and Mercure, E. W. 2007. The pomegranate: a new look at the fruit of paradise. Horticultural Science, 42: 1088-1092.

Wahba, M. L.; Farrag, A. M. I. and Abdel-Hafez, M. A. 1982. Effect of different varieties of grape on the biology of the false mite, Tenuipalpus granati Sayed. Agric. Res. Rev., 60 (1): 323-328.

Zaher, M. A. 1984a. Survey and ecological studies on phytophagous, predaceous and soil mites in Egypt. 1. Phytophagous mites in Egypt (Nile Valley and Delta). PL. 480 Programme U. S. A., Project No. EG-ARS-30, Grant No.FG-EG-139, 228 pp.

Zaher, M. A. 1984b. Survey and ecological studies on phytophagous, predaceous and soil mites in Egypt. III: Mites of Sinai. PL. 480 Programme U. S. A., Project No. EG-ARS-30, Grant No.FG-EG-139, $36 \mathrm{pp}$.

Zaher, M. A. 1986. Survey and ecological studies on phytophagous, predaceous and soil mites in Egypt. II-A: Predaceous \& nonphytophagous mites (Nile Valley and Delta). Text. PL. 480 Programme U. S. A., Project No. EG-ARS-30, Grant No.FG-EG139, 567 pp.

Zaher, M. A. and El-Badry, E. A. 1962. Abundance of mites and insects predaceous on tetranychid and tenuipalpid mites in Giza. Bull. Soc. Ent. Egypt, 46: 429-441.

Zaher, M. A. and El-Badry, E. A. 1964. Survey and population studies on red and false spider mites. Acarologia, Fasc. H.S.: 425-433. In: (1 ${ }^{\text {st }}$ Int. Con. Acarol., Fort Collins, Colorado, U.S.A.).

Zaher, M. A. and Yousef, A. A. 1972. Biology of the false spider mites Tenuipalpus punicae P. \& B. in U.A.R. (Acarina - Tenuipalpidae). Z. ang. Ent., 70: 23-29.

Zaher, M. A.; Wafa, A. K. and Yousef. A. A. 1971. Biology of Brevipalpus phoenicis (Geijskes), in Egypt.Bull. Soc. Ent. Egypte, 54: 177-183.

Zaher, M. A.; Yousef, A. E. A. and Kandil, M. M. 1981. Effect of food on the biology of Cheletogenes ornatus (C.\& F.) (Acari: Prostigmata: Cheyletidae). Acarologia, 22 (4): 361-366.

Zaher, M.A.; Wafa, A.K.; Maher Ali, M. and Rasmy, A.H. 1970. Survey of mites associated with citrus trees in Egypt and Gaza Strip. Bulletin de la Society Entomologique d'Egypte 56: 73-79. 